\title{
Group B Streptococcus and the vaginal microbiome among pregnant women: a systematic review
}

\author{
Sungju Lim $^{1}$, Shilpa Rajagopal ${ }^{2}$, YeRyn Jeong ${ }^{1}$, Dumebi Nzegwu ${ }^{3}$, Michelle Lynn Wright ${ }^{\text {Corresp. 1,4 }}$ \\ ${ }^{1}$ School of Nursing, The University of Texas at Austin, Austin, Texas, United States \\ 2 College of Natural Sciences, Biology Instructional Office, The University of Texas at Austin, Austin, Texas, United States \\ 3 College of Liberal Arts, Department of Health and Society, The University of Texas at Austin, Austin, Texas, United States \\ 4 Dell Medical School, Department of Women's Health, University of Texas at Austin, Austin, Texas, United States \\ Corresponding Author: Michelle Lynn Wright \\ Email address: michelle.wright@utexas.edu
}

Background. Vaginal microbiome studies frequently report diversity metrics and communities of microbiomes associated with reproductive health outcomes. Reports of Streptococcus agalactiae (also known as Group B Streptococcus or GBS), the leading cause of neonatal infectious morbidity and mortality, are notably lacking from the studies of the vaginal microbiome, despite being a known contributor to preterm birth and other complications. Therefore, the purpose of this systematic review was to explore the frequency of GBS reporting in vaginal microbiome literature pertaining to pregnancy and to examine methodological bias that contributes to differences in species and genus-level microbiome reporting. Lack of identification of GBS via sequencing-based approaches due to methodologic or reporting bias may result incomplete understanding of bacterial composition during pregnancy and subsequent birth outcomes.

Methodology. A systematic review was conducted following the PRISMA guideline. Three databases (PubMed, CINAHL, and Web of Science) were used to identify papers for review based on the search terms "vaginal microbiome", "pregnancy", and "16S rRNA sequencing". Articles were evaluated for methods of DNA extraction and sequencing, 16S region, taxonomy classification database, number of participants or vaginal specimens, and pregnancy trimester.

Results. Forty-five research articles reported employing a metagenomic approach or 16S approach for vaginal microbiome analysis during pregnancy that explicitly reported taxonomic composition and were included in this review. Less than $30 \%$ of articles reported the presence of GBS $(\mathrm{N}=13)$. No significant differences in methodology were identified between articles that reported versus did not report GBS. However, there was large variability across research methods used for vaginal microbiome analysis and species-level bacterial community reporting.

Conclusion. Considerable differences in study design and data formatting methods may contribute to underrepresentation of GBS, and other known pathogens, in existing vaginal microbiome literature. Previous studies have identified considerable variation in methodology across vaginal microbiome studies. This study adds to this body of work because in addition to laboratory or statistical methods, how results and data are shared (e.g., only analyzing genus level data or 20 most abundant microbes), may hinder reproducibility and limit our understanding of the influence of less abundant microbes. Sharing detailed methods, analysis code, and raw data may improve reproducibility and ability to more accurately compare microbial communities across studies. 


\section{Title: Group B Streptococcus and the Vaginal}

2 Microbiome among Pregnant Women: A Systematic

3 Review

4

5 Sungju Lim ${ }^{1}$, Shilpa Rajagopal ${ }^{2}$, YeRyn Jeong ${ }^{1}$, Dumebi Nzegwu ${ }^{3}$, Michelle Lynn Wright ${ }^{1,4}$ 6

$7 \quad{ }^{1}$ School of Nursing, The University of Texas at Austin, Austin, Texas, USA

$8{ }^{2}$ College of Natural Sciences, Biology Instructional Office, The University of Texas at Austin, 9 Austin, Texas, USA

$10{ }^{3}$ College of Liberal Arts, Department of Health and Society, The University of Texas at Austin, 11 Austin, Texas, USA

$12{ }^{4}$ Department of Women's Health, Dell Medical School, The University of Texas at Austin,

13 Austin, Texas, USA

15 Corresponding author:

16 Michelle Lynn Wright ${ }^{1,4}$

171710 Red River St. Austin, Texas, 78712,

18 USA Email address: michelle.wright@utexas.edu 
19 Abstract

20 Background. Vaginal microbiome studies frequently report diversity metrics and communities

21 of microbiomes associated with reproductive health outcomes. Reports of Streptococcus

22 agalactiae (also known as Group B Streptococcus or GBS), the leading cause of neonatal

23 infectious morbidity and mortality, are notably lacking from the studies of the vaginal

24 microbiome, despite being a known contributor to preterm birth and other complications.

25 Therefore, the purpose of this systematic review was to explore the frequency of GBS reporting

26 in vaginal microbiome literature pertaining to pregnancy and to examine methodological bias

27 that contributes to differences in species and genus-level microbiome reporting. Lack of

28 identification of GBS via sequencing-based approaches due to methodologic or reporting bias

29 may result incomplete understanding of bacterial composition during pregnancy and subsequent

30 birth outcomes.

31 Methodology. A systematic review was conducted following the PRISMA guideline. Three

32 databases (PubMed, CINAHL, and Web of Science) were used to identify papers for review

33 based on the search terms "vaginal microbiome", "pregnancy", and "16S rRNA sequencing".

34 Articles were evaluated for methods of DNA extraction and sequencing, 16S region, taxonomy

35 classification database, number of participants or vaginal specimens, and pregnancy trimester.

36 Results. Forty-five research articles reported employing a metagenomic approach or $16 \mathrm{~S}$

37 approach for vaginal microbiome analysis during pregnancy that explicitly reported taxonomic

38 composition and were included in this review. Less than $30 \%$ of articles reported the presence of

39 GBS $(\mathrm{N}=13)$. No significant differences in methodology were identified between articles that

40 reported versus did not report GBS. However, there was large variability across research

41 methods used for vaginal microbiome analysis and species-level bacterial community reporting. 
42 Conclusion. Considerable differences in study design and data formatting methods may

43 contribute to underrepresentation of GBS, and other known pathogens, in existing vaginal

44 microbiome literature. Previous studies have identified considerable variation in methodology

45 across vaginal microbiome studies. This study adds to this body of work because in addition to

46 laboratory or statistical methods, how results and data are shared (e.g., only analyzing genus

47 level data or 20 most abundant microbes), may hinder reproducibility and limit our

48 understanding of the influence of less abundant microbes. Sharing detailed methods, analysis

49 code, and raw data may improve reproducibility and ability to more accurately compare

50 microbial communities across studies.

51 Keywords. vaginal microbiome, pregnancy, 16S ribosomal RNA, whole genome sequencing,

52 Streptococcus agalactiae, Group B Streptococcus, GBS 


\section{Introduction}

54 Variations within the vaginal microbiome have been associated with higher risk for preterm birth

55 and other pregnancy complications, such as chorioamnionitis. Microbes associated with these

56 complications such as Streptococcus agalactiae and Gardnerella vaginalis, are frequently

57 present in polymicrobial, as well as Lactobacillus predominant vaginal microbiomes ${ }^{1}$. In light of

58 these considerations, a growing body of research has focused on characterizing the vaginal

59 microbiome using metagenomic approaches. Among the most common methodologies are those

60 that rely on universal targets, 16S ribosomal RNA and cpn60 genes, allowing for more detailed

61 taxonomic classification and profiling of the vaginal microbiome during pregnancy than culture

62 based or targeted sequencing methods.

63 However, data from amplicon-based metagenomic characterizations suggest that such

64 methods provide an incomplete understanding of the species-level diversity present in the

65 vaginal microbiome ${ }^{2}$. For example, there is a notable lack of discussion of S. agalactiae,

66 commonly referred to as Group B Streptococcus (GBS), a known pathogen associated with poor

67 maternal, fetal, and neonatal outcomes in amplicon-based metagenomic vaginal microbiome

68 studies during pregnancy. To date, many vaginal microbiome papers related to birth outcomes

69 primarily focus on differences community state types, which is largely determined by the

70 abundance and type of Lactobacillus present ${ }^{1}$. Focus only on the larger vaginal community state

71 type for analysis may limit evaluation and reporting of microbial species that are known

72 pathogens of pregnancy that are present in low abundance.

73 Currently, in the United States, screening for GBS occurs between 36 to 38 weeks of

74 pregancy $^{3}$, with those who test positive receiving antibiotic treatment during labor to help

75 prevent early-onset neonatal GBS infection. Previous data indicate that approximately one in five 
76 women are colonized with $\mathrm{GBS}^{4}$. Yet, apart from this routine clinical screening near the time of

77 birth, there are limited metagenomic studies that identify or evaluate GBS during pregnancy.

78 While screening for GBS prior to birth has reduced early onset neonatal sepsis caused by GBS in

79 the U.S. ${ }^{5}$, the transient nature of maternal GBS colonization is not well understood ${ }^{6,7}$. Improved

80 understanding of the dynamics of maternal GBS colonization during pregnancy can provide

81 additional insights for preventing GBS complications affecting maternal and fetal health

82 throughout pregnancy and birth (e.g., miscarriage and stillbirth).

83 To our knowledge, there is no systematic review addressing lack of representation of

84 known pathogens, such as GBS, associated with pregnancy complications in existing vaginal

85 microbiome literature. Therefore, this systematic review was conducted to: 1) determine how

86 often GBS is reported in studies of the vaginal microbiome during pregnancy, and 2) analyze the

87 study methods used to identify potential methodological bias in GBS reporting or

88 underrepresentation in vaginal microbiome studies during pregnancy.

89 Methods

90 Protocol and Registration

91 This systematic review was conducted in accordance with the Preferred Reporting Items for

92 Systematic reviews and Meta-analyses (PRISMA) guidelines ${ }^{8}$.

\section{Eligibility Criteria}

94 Original studies that investigated the vaginal microbiome during human pregnancy, were written

95 in English, and published within the past 10 years were included. Only studies with pregnant

96 women as study participants and reported microbiome taxonomy were included. Review papers,

97 editorials, commentaries, and methodological papers including procedure/protocols were 
98 excluded. Research studies that used a targeted approach (i.e.: only on specific microbial taxa,

99 such as Lactobacillus species, were analyzed via microbe specific PCR), were also excluded.

\section{Information Sources}

101 Three electronic databases PubMed, CINAHL, and Web of Science were used for data extraction 102 (date last searched: June 30, 2020).

\section{Search}

104 We searched these databases using two groups of search terms: ("vaginal microbiome" or 105 "vaginal microbiota" or "pregnancy") and ("16S ribosomal RNA" or "16S rRNA" or "whole genome sequencing”). For example, in PubMed, ((((vaginal microbiome) OR (vaginal microbiota))) AND pregnancy) AND ((16s ribosomal rRNA) OR (16s rRNA) OR (whole genome sequencing) OR (metagenomic)) were entered in the search window with the display option 'best match'. Limitations of publication date as '10 years', species as 'humans', and language as 'English' were used (Appendix S1).

\section{Study Selection}

112 After combining articles from the three databases and removing duplicates, five reviewers (SL,

113 SR, YJ, DN, MLW) independently screened all results with titles and abstracts. Disagreements

114 were resolved by discussion among all five reviewers to reach consensus. After the screening, 115 eligibility was checked with full text by five reviewers (SL, SR, YJ, DN, MLW), who each 116 reviewed one fifth of the total articles and cross-reviewed another one fifth. Disagreements were 117 resolved by discussion to reach consensus. 


\section{Data Collection Process}

119 Data was extracted from the included studies by using a collaborative Google Sheets document.

120 Each of five reviewers (SL, SR, YJ, DN, MLW) extracted one fifth of the included studies and

121 cross-checked the accuracy of extracted data from another one fifth. Ambiguity was resolved

122 through discussion among all reviewers.

\section{Data Items}

124 The initial data extraction tool contained the items as follows: study title, study purpose, whether

125 GBS was reported, level of analysis - genus/species (in the case of GBS not reported),

126 sequencing methods, 16S rRNA primer region (for studies using 16S rRNA sequencing),

127 sequencing platform, taxonomy classification database, DNA collection kit, DNA extraction kit,

128 number of pregnant women, number of vaginal specimens, age of pregnant women, pregnancy

129 period in which the specimen collected, ethnicity/region of the participants/study design. In

130 studies that reported taxonomy to the species level and GBS was not detected, we also assessed

131 the articles to determine if Streptococcus at the genus level was reported.

\section{Risk of Bias in Individual Studies}

133 No previous studies have exclusively examined the methodology of vaginal microbiome studies

134 related to detection of known common opportunistic pathogens, like GBS. One of the main

135 purposes of this review was to evaluate if methodological differences/biases in vaginal

136 microbiome studies contribute to reporting or underrepresentation of GBS in existing

137 microbiome literature. 


\section{Summary Measures}

139 Our primary outcome was to identify the number of studies that reported the presence of GBS

140 within the vaginal microbiome. Due to methodological heterogeneity, the differences across

141 analytic and sequencing methodologies between studies that reported versus did not report GBS

142 were compared narratively.

\section{Synthesis of Results}

144 After data extraction, the included studies were divided into two groups: GBS reported and GBS

145 not reported. Independent t-tests were conducted to compare the number of pregnant women and

146 the number of vaginal specimens between GBS reported and not reported groups. The

147 commonalities and differences among 16S rRNA primer regions used were compared. We then

148 qualitatively compared the commonalities and differences of the other methodological categories

149 between two groups, since methods were too varied to compare statistically.

\section{Results}

\section{Study Selection}

152 Our search strategy initially yielded 278 manuscripts (Figure 1). After removing 68 duplicates,

153210 article titles and abstracts were screened, and 159 studies were excluded because they did

154 not meet inclusion criteria. Full text was reviewed for the remaining 52 manuscripts, and 6

155 additional studies were excluded for failing to meet inclusion criteria. A total of 45 studies were

156 included and reviewed in this study. 


\section{Study Characteristics and Results of Individual Studies}

158 The characteristics of each study that reported $(n=13)^{9-21}$ or did not report GBS $(n=32)^{22-53}$ are

159 described in the Supplementary Table $\mathbf{S 1}$ and $\mathbf{S 2}$ respectively.

160 Synthesis of Results

161 Among the 45 studies reviewed, less than $30 \%$ of manuscripts reported the presence of GBS

162 within the results, figures, or microbial taxonomy tables. To determine the frequency in which

163 GBS was reported, and to compare analytic methods across studies, we analyzed: time of sample

164 collection, number of research participants and samples, DNA extraction method, type of

165 sequencing completed, and database used to assign taxonomy (Table 1).

166 Participant and sample characteristics

167 There were no differences in the sample sizes (mean comparison of the number of specimens: $\mathrm{t}=$

$1680.372, \mathrm{df}=43, \mathrm{p}=0.712$; number of women: $\mathrm{t}=0.862, \mathrm{df}=43, \mathrm{p}=0.394)$. However, there was

169 large variability in sample size and number of specimens collected in studies that reported and

170 did not report GBS. Additionally, four manuscripts that did not report GBS within the taxonomy

171 analyzed, or overall metagenomic results, did report positive culture-based GBS clinical

172 screening results for women within the study $32-33,41,44$.

173 DNA extraction kits, Sequencing method, and Taxonomy classification

174 A variety of approaches were employed for DNA extraction; custom developed protocols, along

175 with two standard extraction kits (Table 1) were used in studies reporting GBS within the

176 vaginal microbiome. However, these kits and custom approaches were also used in studies that

177 did not report GBS. In terms of sequencing methods, all studies reporting GBS used 16S rRNA

178 sequencing, as well as most studies that did not report the presence of GBS. Additionally, there

179 was no notable difference found in the 16S rRNA hypervariable region used across either set of 
180 studies. Similarly, multiple taxonomy databases were used for studies that did and did not report 181 the presence of GBS.

\section{Risk of Bias Across Studies}

183 The included studies were not originally conducted with a specific focus on GBS. However, 184 most studies were evaluating the composition of the microbiome across pregnancy, or related to 185 birth outcomes that GBS can influence, such as preterm birth (Supplementary Tables S1 and 186 S2). There is notable bias against GBS reporting when only genus level data are reported. And 187 the practice of only speciating Lactobacillus in vaginal microbiome studies may need to be 188 reconsidered, given GBS cannot be differentiated without also speciating Streptococcus. For 189 example, seven studies that did not report GBS only assigned taxonomy to the genus level, 9 190 studies only assigned species level assignments for Lactobacillus spp., three studies only 191 assigned species to Lactobacillus spp. and Gardnerella, and three studies that did assign 192 taxonomy to species level for analysis did not report species level results (Table S1). Given that 193 species other than Lactobacillus can negatively impact vaginal health and birth outcomes, these 194 methods of analysis and reporting contribute to positive bias towards Lactobacillus reporting in 195 the vaginal microbiome literature and negative bias towards other relevant pathogenic species 196 that are less prevalent. Further, four studies only reported the top 15-21 most abundant species or 197 taxa (Table S1). Since some vaginal pathogens present in low abundance can cause pregnancy 198 complications the underrepresentation of these pathogens, such as GBS, may indicate bias against reporting of microbes that are not the most abundant taxonomic groups but still could be 200 contributing to the poor outcomes being studied. 


\section{Discussion}

203 In this review, we explored how often GBS was reported in studies of the vaginal microbiome

204 during pregnancy. Methods related to GBS reporting were compared to identify potential 205 methodological bias. Among 45 studies, 13 (28.9\%) reported GBS. However, due to the 206 heterogeneity of methods across study approaches, we were not able to detect any systematic 207 differences that appeared to enhance or hinder GBS reporting.

\section{Methodologic differences and sources of bias}

209 There are known sources of bias in metagenomic studies associated with analysis methods ${ }^{3,54-55}$.

210 Brooks and colleagues determined that microbiome community composition can be biased at

211 various steps including DNA extraction, PCR amplification, sequencing, and taxonomic

212 classification ${ }^{54}$. The researchers reported that more than $85 \%$ of the microbiome community

213 results were biased by small variations in methods (less than 5\%). Specifically, it has been

214 determined that variations in DNA extraction and amplification can result in decreased

215 representation of Streptococcus species. If women with GBS colonization only harbor small

216 amounts of the bacteria, it is possible that bias introduced by these steps could have contributed

217 to the limited representation of GBS in vaginal microbiome data.

218 Such underrepresentation of known pathogens can significantly limit the clinical scope of

219 vaginal microbiome studies. For example, many studies only reported results related to

220 community state types or analyzed the most abundant species. Consequently, if thresholds for

221 inclusion are based on sample abundance (e.g., 5\% of sample or 5,000 reads), GBS data may

222 have been excluded if it did not meet the threshold. Furthermore, selection of $16 \mathrm{~S}$ regions for 223 amplification and associated primers can influence genus and species level resolution ${ }^{56}$. For

224 example, it is generally more difficult to distinguish Lactobacillus species within the V4 region, 
225 but may be easier to do so in V1-V3 ${ }^{57}$. Conversely, G. vaginalis is more difficult to detect and 226 differentiate within the V1-V2 region because its sequence is highly variable prior to the V1 227 region, making primer selection more complex ${ }^{56}$. These biases can be partially mitigated by 228 recognizing the characteristics of $16 \mathrm{~S}$ rRNA sequencing and thoughtfully selecting primers.

229 Studies that evaluate the influence of selected 16S regions on GBS representation have not yet 230 been published. From our findings, the 13 studies that detected GBS used 16S regions between 231 V1-V4 or V6. However, the 32 studies that did not report GBS also used the same regions. Thus, 232 assessment of bias by $16 \mathrm{~S}$ region is important when designing and evaluating future vaginal 233 microbiome studies, and further targeted studies evaluating the influence of primer region on 234 known pregnancy pathogens should be completed.

235 Given limitations in exploring the true proportions within the microbial community, 236 studies have increasingly employed whole genome sequencing (WGS), which allows for higher 237 resolution up to the strain level. In our review, two studies employed WGS but did not report $238 \mathrm{GBS}^{43,52}$. Both papers initially selected some of the most frequent species-level taxa, which are 239 frequently Lactobacillus and Gardnerella species, to continue further analysis. This approach 240 may have constrained the strength of WGS, causing researchers to miss less abundant, yet 241 clinically important genus, species, and strains. If WGS was completed to gain a more inclusive 242 representation of all species in the vaginal microbiome, analysis should include more taxa 243 instead of only including the same top taxa that were included in previous $16 \mathrm{~S}$ studies. 244 


\section{Limited assessment and lack of focus on low abundance 246 organisms}

247 The above results indicate that reporting strategies, rather than methodology, may contribute to

248 the underreporting of less abundant pathogens in metagenomic vaginal microbiome studies

249 during pregnancy. As the majority of studies in our review explored the compositional variation

250 of vaginal microbiome during pregnancy, underreporting can be a serious issue because known

251 pathogens may not be analyzed as potential contributors to pregnancy complications.

252

We observed differences in threshold determinants for analysis or reporting. Among the

25332 studies that did not report GBS, 24 analyzed the microbiome at the species level, but only

254 reported the most prevalent genus (i.e., Lactobacillus species). This may lead to a

255 misclassification error, in which only the most abundant bacteria or species is associated with the

256 outcome of interest. Additionally, 20 studies reported Streptococcus at the genus level, but did

257 not conduct further species-level analysis. It is possible that studies that did not speciate

258 Streptococcus had GBS present within their population, a result which could be re-evaluated if

259 raw sequencing data is made available.

260 Moreover, four studies that did not report GBS in their results or microbiome data

261 identified GBS-positive women in their study based on culture-based clinical testing ${ }^{32-33,41,44}$.

262 This suggests that there may be a benefit to using both sequence-based and culture-based

263 methods to verify presence or absence of low abundance microbes within vaginal microbiome

264 communities. Previous studies have shown discordance in culture-based GBS colonization

265 results between antenatal screening and culture on admission for delivery ${ }^{6,58}$. In a study by

266 McCoy and colleagues, nearly $40 \%$ of women that were positive at GBS screening were not

267 GBS positive at the time of admission ${ }^{6}$. Alternatively, Hussain et al. reported a discordance rate 
268 of $11 \%{ }^{58}$. While the transient nature of GBS colonization is known, factors that contribute to loss 269 or gain of maternal GBS colonization remain uncertain 7.

270 Evaluation of culture-based GBS screening previously determined that GBS transmission

271 to neonates is more likely when maternal colonization is heavy, as determined by the number of

272 colonies grown at the time of screening ${ }^{59-60}$. However, it is unclear how well clinical culture-

273 based microbial assessments correlate with the detection of clinically relevant pathogens in

274 sequencing-based vaginal microbiome studies. Furthermore, much of the research investigating

275 risk of neonatal GBS infection related to maternal bacterial load was completed in the 1980s and

276 has not been evaluated using sequencing-based methods ${ }^{60}$. Future studies that concurrently

277 evaluate the accuracy of GBS reporting within microbial communities via metagenomic

278 sequencing approaches and culture-based methods are needed.

\section{Strengths and Limitations}

280 Strengths of this review include the evaluation of factors that may influence the reporting of less

281 abundance vaginal pathogens in existing microbiome literature for a known pathogen that is

282 rarely discussed in the vaginal microbiome literature. We identified studies from multiple

283 databases and assessed differences across studies at various decision points to analyze

284 metagenomic data.

285 The heterogeneity across studies in methods and data reporting limited the ability to pool

286 and re-analyze the data. This was further complicated by the fact that some studies reported most

287 microbial taxa at solely the genus level, whereas other studies only included species-level data

288 for specific genus like Lactobacillus. Furthermore, the format and availability of data associated

289 with manuscripts also varied greatly (e.g., raw data versus including only taxonomy tables used

290 in the analysis). In cases where the full microbial datasets were not available, it was difficult to 
291 determine whether the absence of GBS reporting was due to lack of detection, exclusion from

292 analysis because of low abundance, or some other factor. These limitations highlight the

293 importance of making study analysis code and raw data freely available upon publication in

294 order to encourage reproducibility and comparison.

295

296 Conclusion

297 Only a small proportion of vaginal microbiome studies reported the presence of GBS during 298 pregnancy. No systematic differences in factors previously known to introduce bias in 299 microbiome studies were associated with the lack of GBS reporting. However, there was 300 considerable heterogeneity in research methods employed across studies. Consideration of less 301 abundant, but clinically meaningful microbes in vaginal microbiome studies may improve our 302 understanding of how the vaginal microbiota influences pregnancy outcomes. GBS was reported 303 in studies across all trimesters of pregnancy using metagenomic methods, although reporting of 304 culture based GBS does not always correlate with metagenomic GBS reporting. Therefore, 305 studies evaluating the presence of GBS may need to adopt both confirmatory testing for GBS via 306 culture and parallel comparison with results obtained from metagenomic/meta-taxonomic 307 approaches.

\section{Disclosure of Interests:}

310 None declared. Completed disclosure of interests form available to view online as supporting

311 information. 


\section{Contribution to Authorship}

314 SL, SR and MLW designed and planned the study. SL, SR and MLW performed the search. SL,

315 SR, YJ, DN, and MLW independently screened and selected eligible studies, and completed data

316 extraction, verifying each other's work. SL, SR, YJ and MLW contributed to the interpretation of

317 the data and the writing of the article. All authors reviewed and approved the final version of the

318 manuscript and accepted responsibility for the paper as published.

319

320

321

322

323

324

325

326

327

328

329

330

331

332

333

\section{Details of Ethics Approval}

Not applicable.

\section{Funding}

MLW is supported by the National Institute of Nursing Research (K01NR017903). The content is solely the responsibility of the author and does not necessarily represent the official views of the NIH.

\section{Supporting Information}

Additional supporting information may be found online in the Supporting Information section at the end of the article.

Table S1. Details of the included studies - GBS reported studies $(\mathrm{n}=13)$

Table S2. Details of the included studies - GBS not reported studies $(\mathrm{n}=32)$

Appendix S1. Database search strategy. 


\section{References}

335 1. Brooks JP, Buck GA, Chen G, Diao L, Edwards DJ, Fettweis JM, et al. Changes in

336 vaginal community state types reflect major shifts in the microbiome. Microb Ecol Health

337 Dis [Internet]. 2017;28(1):1303265. Available from:

338 http://www.ncbi.nlm.nih.gov/pubmed/28572753

339 2. Bayar E, Bennett PR, Chan D, Sykes L, MacIntyre DA. The pregnancy microbiome and

340 preterm birth. Semin Immunopathol [Internet]. 2020 Aug;42(4):487-99. Available from:

341 http://www.ncbi.nlm.nih.gov/pubmed/32797272

342 3. Committee Opinion No. 797: Prevention of Group B Streptococcal Early-Onset Disease in

343 Newborns: Correction. Obstet Gynecol [Internet]. 2020;135(4):978-9. Available from:

$344 \quad$ http://www.ncbi.nlm.nih.gov/pubmed/32217968

345 4. Russell NJ, Seale AC, O’Driscoll M, et al. Maternal Colonization With Group B

346 Streptococcus and Serotype Distribution Worldwide: Systematic Review and Meta-

347 analyses. Clin Infect Dis. 2017;65(suppl_2):S100-S111. doi:10.1093/cid/cix658

348 5. Phares CR, Lynfield R, Farley MM, Mohle-Boetani J, Harrison LH, Petit S, et al.

349 Epidemiology of invasive group B streptococcal disease in the United States, 1999-2005.

350 JAMA [Internet]. 2008 May 7;299(17):2056-65. Available from:

351 http://www.ncbi.nlm.nih.gov/pubmed/18460666

352 6. McCoy JA, Elovitz MA, Alby K, Koelper NC, Nissim I, Levine LD. Association of

353 Obesity With Maternal and Cord Blood Penicillin Levels in Women With Group B

354 Streptococcus Colonization. Obstet Gynecol [Internet]. 2020 Oct;136(4):756-64.

355 Available from: http://www.ncbi.nlm.nih.gov/pubmed/32925625

356 7. Hansen SM, Uldbjerg N, Kilian M, Sørensen UBS. Dynamics of Streptococcus agalactiae 
357 colonization in women during and after pregnancy and in their infants. J Clin Microbiol 358 [Internet]. 2004 Jan;42(1):83-9. Available from:

359 http://www.ncbi.nlm.nih.gov/pubmed/14715736

360 8. Moher D, Liberati A, Tetzlaff J, Altman DG, Altman D, Antes G, et al. Preferred 361 reporting items for systematic reviews and meta-analyses: The PRISMA statement. PLoS $362 \quad$ Med. 2009;6(7).

363 9. Fettweis JM, Serrano MG, Huang B, Brooks JP, Glascock AL, Sheth NU, et al. An 364 emerging mycoplasma associated with trichomoniasis, vaginal infection and disease.

$365 \quad$ PLoS One. 2014;9(10).

366 10. Ghartey JP, Smith BC, Chen Z, Buckley N, Lo Y, Ratner AJ, et al. Lactobacillus crispatus 367 dominant vaginal microbiome is associated with inhibitory activity of female genital tract 368 secretions against Escherichia coli. PLoS One. 2014;9(5):1-8.

369 11. Romero R, Hassan SS, Gajer P, Tarca AL, Fadrosh DW, Bieda J, et al. The Vaginal 370 microbiota of pregnant women who subsequently have spontaneuos preterm labor and delivery and those with a normal delivery at term. Microbiome. 2014;1-15.

372 12. Romero R, Hassan SS, Gajer P, Tarca AL, Fadrosh DW, Nikita L, et al. The composition 373 and stability of the vaginal microbiota of normal pregnant women is different from that of non-pregnant women. Microbiome. 2014;2(1):1-19.

375 13. Bisanz JE, Enos MK, PrayGod G, Seney S, Macklaim JM, Chilton S, et al. Microbiota at 376 multiple body sites during pregnancy in a rural tanzanian population and effects of Moringa-supplemented probiotic yogurt. Appl Environ Microbiol. 2015;81(15):4965-75.

378 14. MacIntyre DA, Chandiramani M, Lee YS, Kindinger L, Smith A, Angelopoulos N, et al. 379 The vaginal microbiome during pregnancy and the postpartum period in a European 
380

381

382

383

384

385

386

387

388

389

390

391

392

393

394

395

396

397

398

399

400

401

402

population. Sci Rep [Internet]. 2015 Aug 11;5(1):8988. Available from:

http://www.nature.com/articles/srep08988

15. Kindinger LM, Bennett PR, Lee YS, Marchesi JR, Smith A, Cacciatore S, et al. The interaction between vaginal microbiota, cervical length, and vaginal progesterone treatment for preterm birth risk. Microbiome [Internet]. 2017;5(1):1-14. Available from: http://dx.doi.org/10.1186/s40168-016-0223-9

16. Brown RG, Marchesi JR, Lee YS, Smith A, Lehne B, Kindinger LM, et al. Vaginal dysbiosis increases risk of preterm fetal membrane rupture, neonatal sepsis and is exacerbated by erythromycin. BMC Med. 2018;16(1):1-15.

17. Hočevar K, Maver A, Vidmar Šimic M, Hodžić A, Haslberger A, Premru Seršen T, et al. Vaginal Microbiome Signature Is Associated With Spontaneous Preterm Delivery. Front Med. 2019;6(September):1-12.

18. Das Purkayastha S, Bhattacharya MK, Prasad HK, Upadhyaya H, Lala S Das, Pal K, et al. Contrasting diversity of vaginal lactobacilli among the females of Northeast India. BMC Microbiol. 2019;19(1):1-10.

19. Romero R, Gomez-Lopez N, Winters AD, Jung E, Shaman M, Bieda J, et al. Evidence that intra-amniotic infections are often the result of an ascending invasion - A molecular microbiological study. J Perinat Med [Internet]. 2019 Nov 1 [cited 2020 Sep 5];47(9):915-31. Available from: https://doi.org/10.1515/jpm-2019-0297

20. Tabatabaei N, Eren AM, Barreiro LB, Yotova V, Dumaine A, Allard C, et al. Vaginal microbiome in early pregnancy and subsequent risk of spontaneous preterm birth: a casecontrol study. BJOG An Int J Obstet Gynaecol. 2019;126(3):349-58.

21. Al-Memar M, Bobdiwala S, Fourie H, Mannino R, Lee YS, Smith A, et al. The 
403

404

405

406

407

408

409

410

411

412

413

414

415

416

417

418

419

420

421

422

423

424

425

association between vaginal bacterial composition and miscarriage: a nested case-control study. BJOG An Int J Obstet Gynaecol. 2020;127(2):264-74.

22. Dominguez-Bello MG, Costello EK, Contreras M, Magris M, Hidalgo G, Fierer N, et al. Delivery mode shapes the acquisition and structure of the initial microbiota across multiple body habitats in newborns. Proc Natl Acad Sci U S A. 2010;107(26):11971-5.

23. Hernández-Rodríguez C, Romero-González R, Albani-Campanario M, Figueroa-Damián R, Meraz-Cruz N, Hernández-Guerrero C. Vaginal microbiota of healthy pregnant mexican women is constituted by four Lactobacillus species and several vaginosisassociated bacteria. Infect Dis Obstet Gynecol. 2011;2011.

24. Aagaard K, Riehle K, Ma J, Segata N, Mistretta TA, Coarfa C, et al. A metagenomic approach to characterization of the vaginal microbiome signature in pregnancy. PLoS One. $2012 ; 7(6)$.

25. Frank DN, Manigart O, Leroy V, Meda N, Valéa D, Zhang W, et al. Altered vaginal microbiota are associated with perinatal mother-to-child transmission of HIV in African women from Burkina Faso. J Acquir Immune Defic Syndr. 2012;60(3):299-306.

26. Hyman RW, Herndon CN, Jiang H, Palm C, Fukushima M, Bernstein D, et al. The dynamics of the vaginal microbiome during infertility therapy with in vitro fertilizationembryo transfer. J Assist Reprod Genet [Internet]. 2012 Feb 6;29(2):105-15. Available from: http://link.springer.com/10.1007/s10815-011-9694-6

27. Hyman RW, Fukushima M, Jiang H, Fung E, Rand L, Johnson B, et al. Diversity of the Vaginal Microbiome Correlates With Preterm Birth. Reprod Sci [Internet]. 2014 Jan 28;21(1):32-40. Available from:

http://journals.sagepub.com/doi/10.1177/1933719113488838 
426 28. Walther-António MRS, Jeraldo P, Berg Miller ME, Yeoman CJ, Nelson KE, Wilson BA,

427 et al. Pregnancy's stronghold on the vaginal microbiome. PLoS One. 2014;9(6):1-10.

428 29. Baldwin EA, Walther-Antonio M, MacLean AM, Gohl DM, Beckman KB, Chen J, et al.

429 Persistent microbial dysbiosis in preterm premature rupture of membranes from onset

$430 \quad$ until delivery. PeerJ. 2015;2015(11):1-18.

431 30. DiGiulio DB, Callahan BJ, McMurdie PJ, Costello EK, Lyell DJ, Robaczewska A, et al.

432 Temporal and spatial variation of the human microbiota during pregnancy. Proc Natl Acad

$433 \quad$ Sci U S A. 2015;112(35):11060-5.

434 31. Huang Y-E, Wang Y, He Y, Ji Y, Wang L-P, Sheng H-F, et al. Homogeneity of the

435 Vaginal Microbiome at the Cervix, Posterior Fornix, and Vaginal Canal in Pregnant

436 Chinese Women. Microb Ecol [Internet]. 2015 Feb 18;69(2):407-14. Available from:

437 http://link.springer.com/10.1007/s00248-014-0487-1

438 32. Brumbaugh DE, Arruda J, Robbins K, Ir D, Santorico SA, Robertson CE, et al. Mode of

439 delivery determines neonatal pharyngeal bacterial composition and early intestinal

440 colonization. J Pediatr Gastroenterol Nutr. 2016;63(3):320-8.

441 33. Jayaprakash TP, Wagner EC, Van Schalkwyk J, Albert AYK, Hill JE, Money DM, et al.

442 High diversity and variability in the vaginal microbiome in women following Preterm

443 Premature Rupture of Membranes (PPROM): A prospective cohort study. PLoS One.

2016;11(11):1-19.

445 34. Lauder AP, Roche AM, Sherrill-Mix S, Bailey A, Laughlin AL, Bittinger K, et al.

446 Comparison of placenta samples with contamination controls does not provide evidence

447 for a distinct placenta microbiota. Microbiome [Internet]. 2016;4:1-11. Available from:

$448 \quad$ http://dx.doi.org/10.1186/s40168-016-0172-3 
449 35. Nelson DB, Shin H, Wu J, Dominguez-Bello MG. The Gestational Vaginal Microbiome 450 and Spontaneous Preterm Birth among Nulliparous African American Women. Am J $451 \quad$ Perinatol. 2016;33(9):887-93.

452 36. Subramaniam A, Kumar R, Cliver SP, Zhi D, Szychowski JM, Abramovici A, et al. 453 Vaginal Microbiota in Pregnancy: Evaluation Based on Vaginal Flora, Birth Outcome, and Race. Am J Perinatol. 2016;33(4):401-8.

37. Callahan BJ, DiGiulio DB, Aliaga Goltsman DS, Sun CL, Costello EK, Jeganathan P, et al. Replication and refinement of a vaginal microbial signature of preterm birth in two racially distinct cohorts of US women. Proc Natl Acad Sci U S A. 2017;114(37):9966-71.

38. Freitas AC, Chaban B, Bocking A, Rocco M, Yang S, Hill JE, et al. The vaginal microbiome of pregnant women is less rich and diverse, with lower prevalence of Mollicutes, compared to non-pregnant women. Sci Rep. 2017;7(1):1-16.

39. Nasioudis D, Forney LJ, Schneider GM, Gliniewicz K, France M, Boester A, et al. Influence of Pregnancy History on the Vaginal Microbiome of Pregnant Women in their First Trimester. Sci Rep. 2017;7(1):1-6.

40. Nasioudis D, Forney LJ, Schneider GM, Gliniewicz K, France MT, Boester A, et al. The composition of the vaginal microbiome in first trimester pregnant women influences the level of autophagy and stress in vaginal epithelial cells. J Reprod Immunol [Internet]. 2017;123(March):35-9. Available from: http://dx.doi.org/10.1016/i.jri.2017.08.009

41. Roesch LFW, Silveira RC, Corso AL, Dobbler PT, Mai V, Rojas BS, et al. Diversity and composition of vaginal microbiota of pregnant women at risk for transmitting Group B Streptococcus treated with intrapartum penicillin. PLoS One. 2017;12(2):1-13. 
472

473

474

475

476

477

478

479

480

481

482

483

484

485

486

487

488

489

490

491

492

493

494

microbiome trends and preterm birth. Am J Obstet Gynecol. 2017;217(3):356.e1-356.e18.

43. Goltsman DSA, Sun CL, Proctor DM, DiGiulio DB, Robaczewska A, Thomas BC, et al. Metagenomic analysis with strain-level resolution reveals fine-scale variation in the human pregnancy microbiome. Genome Res. 2018;28(10):1467-80.

44. Leizer J, Nasioudis D, Forney LJ, Schneider GM, Gliniewicz K, Boester A, et al. Properties of Epithelial Cells and Vaginal Secretions in Pregnant Women When Lactobacillus crispatus or Lactobacillus iners Dominate the Vaginal Microbiome. Reprod Sci. 2018;25(6):854-60.

45. Matsumoto A, Yamagishi Y, Miyamoto K, Oka K, Takahashi M, Mikamo H. Characterization of the vaginal microbiota of Japanese women. Anaerobe [Internet]. 2018;54:172-7. Available from: https://doi.org/10.1016/j.anaerobe.2018.10.001

46. Wylie KM, Blankenship SA, Tuuli MG, Macones GA, Stout MJ. Evaluation of patientversus provider-collected vaginal swabs for microbiome analysis during pregnancy. BMC Res Notes. 2018;11(1):5-11.

47. Chen Y, Hong Z, Wang W, Gu L, Gao H, Qiu L, et al. Association between the vaginal microbiome and high-risk human papillomavirus infection in pregnant Chinese women. BMC Infect Dis. 2019;19(1):1-11.

48. Dobbler P, Mai V, Procianoy RS, Silveira RC, Corso AL, Roesch LFW. The vaginal microbial communities of healthy expectant Brazilian mothers and its correlation with the newborn's gut colonization. World J Microbiol Biotechnol [Internet]. 2019;35(10):1-14. Available from: https://doi.org/10.1007/s11274-019-2737-3

49. He Y, Huang Y, Zhang Z, Yu F, Zheng Y. Exploring profile and potential influencers of vaginal microbiome among asymptomatic pregnant Chinese women. PeerJ. 
495

496

497

498

499

500

501

502

503

504

505

506

507

508

509

510

511

512

513

514

515

516

517

2019;2019(12):1-17.

50. Jefferson KK, Parikh HI, Garcia EM, Edwards DJ, Serrano MG, Hewison M, et al. Relationship between vitamin D status and the vaginal microbiome during pregnancy. J Perinatol. 2019;39(6):824-36.

51. Liu CJ, Liang X, Niu ZY, Jin Q, Zeng XQ, Wang WX, et al. Is the delivery mode a critical factor for the microbial communities in the meconium? EBioMedicine [Internet]. 2019;49:354-63. Available from: https://doi.org/10.1016/j.ebiom.2019.10.045

52. Price JT, Vwalika B, Hobbs M, Nelson JAE, Stringer EM, Zou F, et al. Highly diverse anaerobe-predominant vaginal microbiota among HIV-infected pregnant women in Zambia. PLoS One. 2019;14(10):1-17.

53. Witkin SS, Nasioudis D, Leizer J, Minis E, Boester A, Forney LJ. Epigenetics and the vaginal microbiome: Influence of the microbiota on the histone deacetylase level in vaginal epithelial cells from pregnant women. Minerva Ginecol. 2019;71(2):171-5.

54. Brooks JP, Edwards DJ, Harwich MD, Rivera MC, Fettweis JM, Serrano MG, et al. The truth about metagenomics: Quantifying and counteracting bias in 16S rRNA studies Ecological and evolutionary microbiology. BMC Microbiol. 2015;15(1):1-14.

55. Knight R, Vrbanac A, Taylor BC, Aksenov A, Callewaert C, Debelius J, et al. Best practices for analysing microbiomes. Nat Rev Microbiol [Internet]. 2018;16(7):410-22. Available from: http://dx.doi.org/10.1038/s41579-018-0029-9

56. Graspeuntner S, Loeper N, Künzel S, Baines JF, Rupp J. Selection of validated hypervariable regions is crucial in $16 \mathrm{~S}$-based microbiota studies of the female genital tract. Sci Rep [Internet]. 2018;8(1):9678. Available from:

http://www.ncbi.nlm.nih.gov/pubmed/29946153 
518 57. Fettweis JM, Serrano MG, Sheth NU, Mayer CM, Glascock AL, Brooks JP, et al. Species519 level classification of the vaginal microbiome. BMC Genomics [Internet]. 2012;13 Suppl 520 8:S17. Available from: http://www.ncbi.nlm.nih.gov/pubmed/23282177

521 58. Hussain FN, Al-Ibraheemi Z, Pan S, Francis AP, Taylor D, Lam MC, et al. The Accuracy 522 of Group Beta Streptococcus Rectovaginal Cultures at 35 to 37 Weeks of Gestation in Predicting Colonization Intrapartum. AJP Rep [Internet]. 2019 Jul;9(3):e302-9. Available from: http://www.ncbi.nlm.nih.gov/pubmed/31555492

525

526

527

528

529

530

531

532

59. Berardi A, Rossi C, Guidotti I, Vellani G, Lugli L, Bacchi Reggiani ML, et al. Factors associated with intrapartum transmission of group B Streptococcus. Pediatr Infect Dis J [Internet]. 2014 Dec;33(12):1211-5. Available from:

http://www.ncbi.nlm.nih.gov/pubmed/25037035

60. Seedat F, Brown CS, Stinton C, Patterson J, Geppert J, Freeman K, et al. Bacterial Load and Molecular Markers Associated With Early-onset Group B Streptococcus: A Systematic Review and Meta-analysis. Pediatr Infect Dis J [Internet]. 2018;37(12):e30614. Available from: http://www.ncbi.nlm.nih.gov/pubmed/29601454 
Figure 1

Figure 1. The Flow Diagram of This Study 
Figure 1. The Flow Diagram of This Study

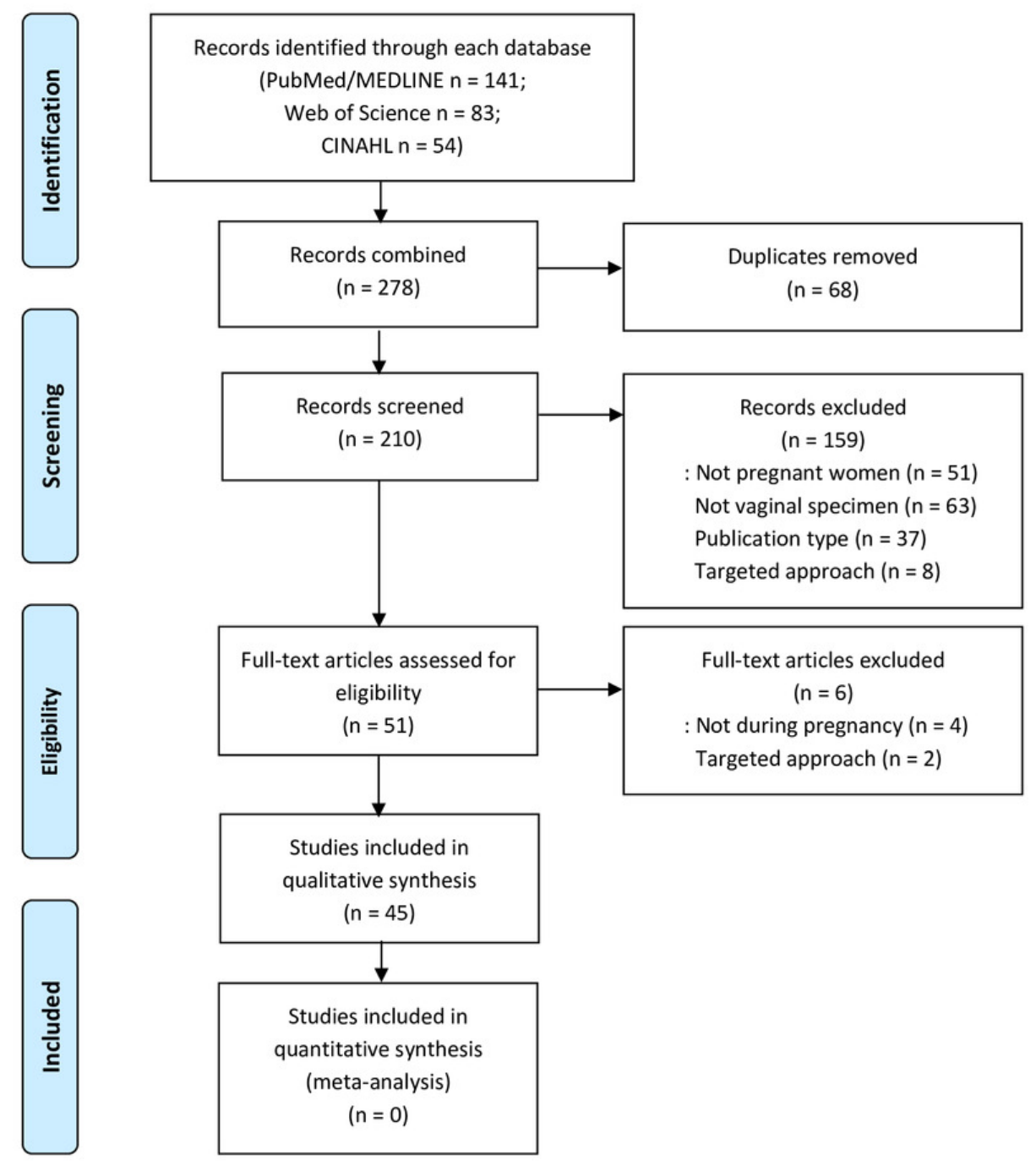




\section{Table 1 (on next page)}

Table 1. Comparison of GBS Reported and GBS Not Reported Studies 
1 Table 1. Comparison of GBS Reported and GBS Not Reported Studies

\begin{tabular}{|c|c|c|}
\hline & GBS Reported $(n=13)$ & GBS Not Reported $(n=32)$ \\
\hline Trimesters & $\begin{array}{l}1^{\text {st }} \text { trimester }(1) \\
2^{\text {nd }} \text { trimester }(0) \\
3^{\text {rd }} \text { trimester }(3) \\
\text { All trimesters }(5) \\
2^{\text {nd }} \text { and } 3^{\text {rd }}(2) \\
\text { Unspecified }(2)\end{array}$ & $\begin{array}{l}1^{\text {st }} \text { trimester }(5) \\
2^{\text {nd }} \text { trimester }(6) \\
3^{\text {rd }} \text { trimester }(6) \\
\text { All trimesters }(12) \\
1^{\text {st }} \text { and } 2^{\text {nd }}(2) \\
2^{\text {nd }} \text { and } 3^{\text {rd }}(5) \\
\text { Unspecified (1) }\end{array}$ \\
\hline $\begin{array}{l}\text { Number of } \\
\text { pregnant } \\
\text { women }\end{array}$ & $\begin{array}{l}\text { Mean: } 111.154 \text { (SD 121.817) } \\
\text { Range [8 - 450], Total \# } 1445\end{array}$ & $\begin{array}{l}\text { Mean: } 82.594 \text { (SD 91.331) } \\
\text { Range [6 - 461], Total \# } 2643\end{array}$ \\
\hline $\begin{array}{l}\text { Number of } \\
\text { vaginal } \\
\text { specimens }\end{array}$ & $\begin{array}{l}\text { (11/13 studies; } 2 \text { unspecified) } \\
\text { Mean: } 184 \\
\text { Standard deviation: } 155.793 \\
\text { Range: }[8-450] \\
\text { Total \#: around } 2086\end{array}$ & $\begin{array}{l}\text { (32/32 studies) } \\
\text { Mean: } 214 \\
\text { Standard deviation: } 418.706 \\
\text { Range: [4 - 2179] } \\
\text { Total \#: around } 6848\end{array}$ \\
\hline $\begin{array}{l}\text { DNA } \\
\text { extraction } \\
\text { kit }\end{array}$ & $\begin{array}{l}\text { QIAamp DNA Mini Kit (6) } \\
\text { MoBio PowerSoil Kit (3) } \\
\text { Custom (4) }\end{array}$ & $\begin{array}{l}\text { QIAamp (6) } \\
\text { MoBio (13) } \\
\text { DNeasy (2) } \\
\text { Others (5) } \\
\text { Custom (5) }\end{array}$ \\
\hline $\begin{array}{l}\text { Sequencing } \\
\text { method }\end{array}$ & 16S rRNA Sequencing (13) & $\begin{array}{l}\text { 16S rRNA Sequencing (29) } \\
\text { Shotgun sequencing (2) } \\
\text { Cpn60 (2) }\end{array}$ \\
\hline $\begin{array}{l}16 \mathrm{~S} \text { rRNA } \\
\text { region }\end{array}$ & $\begin{array}{l}\text { V1-V2 (4), V1-V3 (3), V3-V4 } \\
\text { (1), V4 (3), V6 (1), full (1) }\end{array}$ & $\begin{array}{l}\text { V1-V2 (2), V1-V3 (8), V2 (1), } \\
\text { V3 (1), V3-V4 (3), V3-V5 (7), } \\
\text { V4 (5), V4-V5 (1), V4-V6 (1), } \\
\text { full (6) }\end{array}$ \\
\hline
\end{tabular}

2 